\title{
CONTAMINACIÓN DEL SUELO EN LA ZONA MINERA DE RASGATÁ BAJO (TAUSA). MODELO CONCEPTUAL
}

\section{CONCEPTUAL MODEL OF SOIL POLLUTION IN THE MINING ZONE OF RASGATÁ BAJA (TAUSA)}

María Eugenia Guerrero Useda', Vanessa Pineda Acevedo²

Fecha de recepción: 13 de mayo de 2015

Fecha de aprobación: 29 de febrero de 2016

Referencia: M. E. Guerrero Useda, V. Pineda Acevedo. (2016). Contaminación del suelo en la zona minera de Rasgatá Bajo (Tausa). Modelo conceptual. Ciencia e Ingeniería Neogranadina, 26 (1), pp. 57-74, DOl: http://dx.doi.org/10.18359/ rcin. 1664

\section{RESUMEN}

Se desarrolló un modelo conceptual del riesgo de contaminación físico química del suelo en la vereda Rasgatá Bajo. Esta vereda del municipio de Tausa, en Cundinamarca, está en la zona limítrofe del declarado por la Corporación Autónoma Regional de Cundinamarca en 2009, Distrito Regional de Manejo Integrado de los Recursos Naturales Renovables Páramo Guargua y Laguna Verde. La vocación productiva local por tradición ha sido el cultivo de papa (Solanum tuberosum L) y la extracción y transformación de arcilla y carbón, actividades con graves impactos sobre el suelo, por lo que el objetivo del proyecto fue identificar y evaluar la condición de este recurso en territorios donde confluyen actividades de extracción y transformación de arcilla y carbón. Se siguió un enfoque metodológico mixto que combinó investigación documental, observaciones in situ y medición de características físico químicas para determinar las fuentes y tipos de contaminantes con riesgo de afectar el suelo aledaño a las faenas mineras y con impactos toxicológicos para las personas, la fauna y la flora. Los datos obtenidos aportarán al rediseño y evaluación de los planes de manejo ambiental local.

Palabras clave: contaminación física y química del suelo, degradación físico química del suelo, deterioro estético del suelo, modelo conceptual de contaminación, riesgo de contaminación.

1. Física, PhD en Ciencias Físicas y Matemáticas, Docente, Universidad El Bosque, Bogotá, Colombia, mguerrerou@unbosque.edu.co

2. Ing. Ambiental, Programa de Ingeniería Ambiental de la Universidad El Bosque, Bogotá, Colombia, gpineda@unbosque.edu.co 


\section{ABSTRACT}

A conceptual model of the risk of soil contamination in Rasgatá Bajo was developed. This village in the municipality of Tausa (Cundinamarca) is on the border declared by the Regional Autonomous Corporation of Cundinamarca in 2009, Regional District Integrated Management of Renewable Natural Resources Guargua and Laguna Verde Paramus. The local productive vocation has traditionally been cultivating potato (Solanum tuberosum L) and the extraction and processing of clay and coal, activities with serious impacts on soil, so that the project objective was to identify and evaluate the condition of this resource in areas confluence mining and processing of clay and coal. A mixed methodological approach that combined documentary research, in situ measurement of chemical and physical characteristics remarks followed to determine the sources and types of hazardous pollutants affecting soil adjacent to mine sites and toxicological impacts for people, wildlife and flora. The data obtained will contribute to the redesign and evaluation of local environmental management plans.

Keywords: physical and chemical soil contamination, physicochemical soil degradation, aesthetic deterioration of soil, conceptual model of contamination, contamination risk.

\section{INTRODUCCIÓN}

El suelo es un recurso esencial, del cual depende la salud de las personas, las especies animales y las plantas que lo habitan, así como las potencialidades del desarrollo económico y social de las comunidades que se benefician de este. Y aunque la Carta Política colombiana de 1991 reconoció el valor del suelo, la tímida inserción de este tema en la agenda pública local conllevó a la persistencia de vacíos normativos que hoy dificultan tanto el control público sobre la protección y preservación de este recurso, como la implementación sistemática de acciones de monitoreo y evaluación de la calidad del suelo y de sus impactos sobre la salud de los habitantes y la economía regional, para lo cual en el contexto internacional se han validado sendos modelos matemáticos [1] que están en mora de ser apropiados, incluso en contextos productivos donde el peligro de afectación del recurso suelo es alto [2].
Así, el Estado colombiano, en el literal $a$ del artículo octavo del Decreto Ley 2811 de 1974, definió la contaminación como la alteración del ambiente con sustancias o formas de energía puestas en este por actividad humana o de la naturaleza, en cantidades, concentraciones o niveles capaces de interferir el bienestar y la salud de las personas, atentar contra la flora y la fauna, degradar la calidad del ambiente. Dos actividades humanas que provocan contaminación química y cambios físicos en el suelo son la agricultura y la minería, las que por años confluyeron en varios municipios de Cundinamarca, por lo que es imperativo determinar los contaminantes que podrían haber generado la degradación física y química del suelo y haberse acumulado en concentraciones tóxicas para las personas, la fauna y la flora. La información que resultó de este estudio, además de aportar al diseño de mapas de riesgo, cualifica el diseño y evaluación de los planes de manejo ambiental de ecosistemas estratégicos. 


\section{MATERIALES Y MÉTODOS}

Siguiendo los esquemas metodológicos aplicados en $[3,4]$ se diseñó un estudio de tipo mixto que combinó técnicas cualitativas y cuantitativas para obtener los datos que alimentaron el diseño del modelo conceptual de un suelo posiblemente degradado por contaminantes derivados de actividades antrópicas: extracción y transformación de carbón y arcilla.

El proyecto se organizó y ejecutó por fases según tipos de análisis, como se presenta en el Esquema 1. La primera fase consistió en una investigación documental que aportó datos para construir la descripción del entorno; esta se focalizó en tres aspectos: político, histórico y físico. La siguiente fase inició con una revisión documental sobre técnicas, normas y estándares nacionales e internacionales para el análisis de características físico químicas del suelo, siguió con el diseño de un muestreo al azar de suelos localizados en áreas de influencia agrícola e industrial [6] y finalizó con la toma de muestras y determinación de características físicas del suelo $[7,8]$. Finalmente, se diseñó un modelo conceptual preliminar, que tras ser sometido al análisis de expertos condujo al modelo actualizado [3]. Con los datos obtenidos en los diferentes estudios se alimentó una matriz de amenazas que relacionó, para cada actividad antrópica objeto del estudio, los eventos adversos con impactos de naturaleza física y química sobre el suelo, y su frecuencia de ocurrencia.

Para establecer la concentración de contaminantes químicos y sus vías de movilización es necesario conocer varios

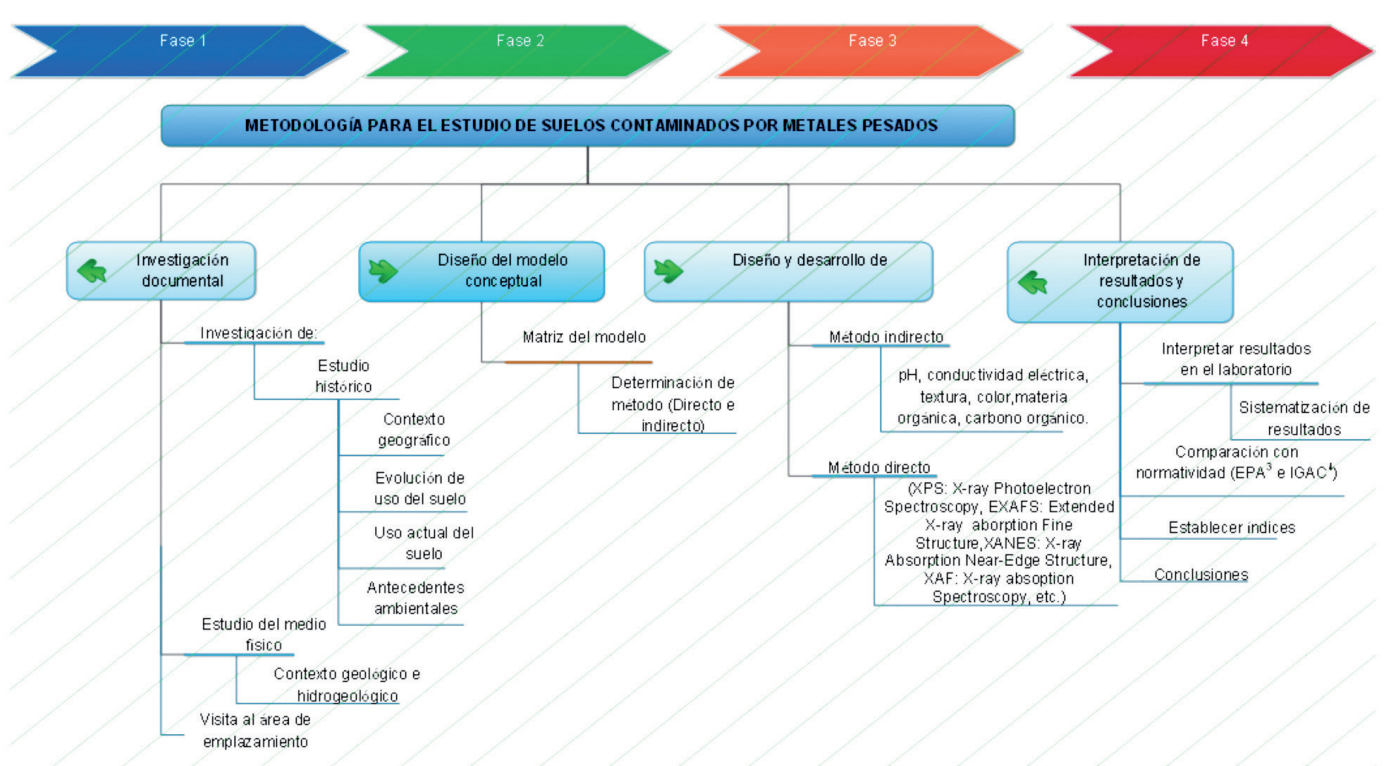

Esquema 1. Diseño metodológico aplicado para la elaboración del modelo conceptual de un suelo posiblemente contaminado por actividades antrópicas.

Fuente: Pineda Acevedo, 2015 [5]. 
parámetros del suelo. Se diseñó y desarrolló un conjunto básico de ensayos analíticos, según normas técnicas colombianas, para determinar la calidad del suelo: toma de muestras para comprobar contaminación (NTC 3656), determinación del pH (NTC 5264), conductividad eléctrica (NTC 5596), determinación de carbono orgánico (NTC 5403) y análisis de espectroscopia para verificar presencia de contaminantes químicos [4]. Los resultados obtenidos se compararon con parámetros EPA.

\section{DESCRIPCIÓN DEL SITIO DE ESTUDIO}

El municipio de Tausa alberga un sistema hidrográfico compuesto por cinco subcuencas de los ríos Singuatoque (Salitre), Cubillos, Checua, Suta y Negro; que a su vez están conformadas por trece microcuencas, sistema al que pertenecen las lagunas Verde y Seca, y páramos aledaños con reservas de agua [9]. En el páramo de la laguna Verde confluyen las divisorias de cuencas del río Bogotá Neusa, Ubaté - Fúquene y Negro (Figura 1), y

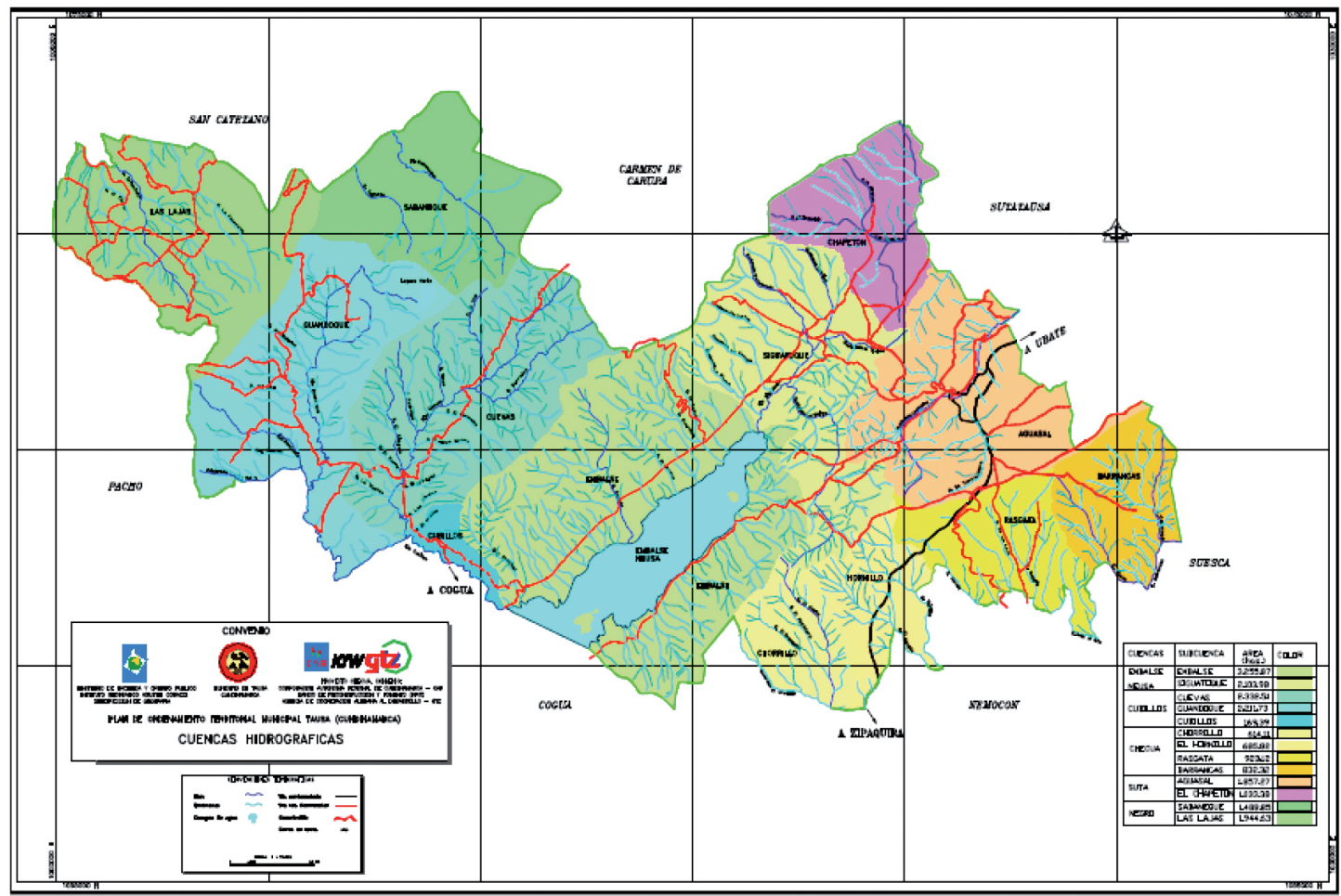

Figura 1. Mapa de las cuencas hidrográficas del municipio de Tausa

Fuente: Instituto Geográfico Agustin Codazi, 1997 [10]. 
aunque los gobiernos departamentales han reconocido el potencial hidrológico de Tausa, pocos proyectos públicos se han dirigido al estudio y recuperación de sus cuencas, salvo por un detallado estudio de 1998, que buscó controlar la erosión de la cuenca del río Checua que recibe el impacto tanto de cultivos de papa Solanum tuberosum $L$ y pastos mejorados, como de la extracción y trasformación de arcillas y carbón [11].

Como ya se señaló, la minería es una actividad ampliamente desarrollada en el municipio de Tausa, que cuenta con 53 títulos mineros, donde la fiscalización del 2014 arrojó que el $75 \%$ de los títulos presentó incumplimientos, con un promedio de 6,6 inconformidades por título, estando el $69 \%$ de estas en títulos en etapa contractual de explotación [12]. En cuanto a la agricultura, debe reiterarse que los principales cultivos son la papa y los pastos mejorados, que se producen con tecnologías tradicionales, semimecanizadas y con uso intensivo de agroquímicos [13].

El Plan de Desarrollo 2012-2015 de Tausa en el análisis de riesgos reportó, en relación con las fuentes hídricas, los siguientes riesgos: la reducción de los caudales de los ríos y quebradas del municipio a causa de la degradación de la parte alta de la cuenca, la contaminación química y biológica de todo el curso de la corriente de las subcuencas de las quebradas Rasgatá, Barrancas, Chorrillo y Hatillo, la degradación y contaminación y, finalmente, la ausencia de planes de manejo de este recurso [13]. Frente al recurso suelo, el diagnóstico no fue tan detallado, pero indicó amenazas de deforestación y erosión severa con factores de vulnerabilidad asociados a la obsolescencia tecnológica de los hornos procesadores de arcilla y de coquización de carbón y al uso de tecnologías inadecuadas para la explotación minera que, según se registra en la Figura 2, se extiende por todo el municipio.

Rasgatá Bajo es una de las 15 veredas del área rural del municipio de Tausa. Con 115 habitantes y 19 viviendas en un área de $4.872 \mathrm{~km}^{2}$, es la segunda vereda menos poblada de Tausa, en la que se concentran empresas ladrilleras y mineras [15] generadoras de contaminantes a los que, de tiempo atrás, se atribuyen la mayoría de los casos de infección respiratoria aguda, primera causa de mortalidad infantil de la zona [16]. Al impacto de la actividad ladrillera y minera en la salud de los habitantes de Rasgatá Bajo se encontró referencia en el Plan de Desarrollo 2008-2011 del municipio, donde se señaló que los productores de ladrillo tienen problemas de salud por la contaminación ambiental asociada a los chircales, que si dejan de funcionar generarían una gran problemática social, por la falta de puestos alternativos de trabajo [17].

\section{RESULTADOS}

Se determinaron como fuentes primarias de contaminación del suelo en la vereda Rasgatá Bajo las siguientes actividades: explotación en minas subterráneas de carbón coquizable, carbón térmico y carbón mineral triturado, explotación a cielo abierto de arcilla común, fabricación de ladrillo en hornos de combustión completa de carbón y coquización de carbón.

Después de establecer las actividades productivas desarrolladas en Rasgatá Bajo, se buscó literatura científica sobre eventos de contaminación del recurso suelo asociados a tales actividades. Primero se revisaron estudios locales «municipio, departamento, 


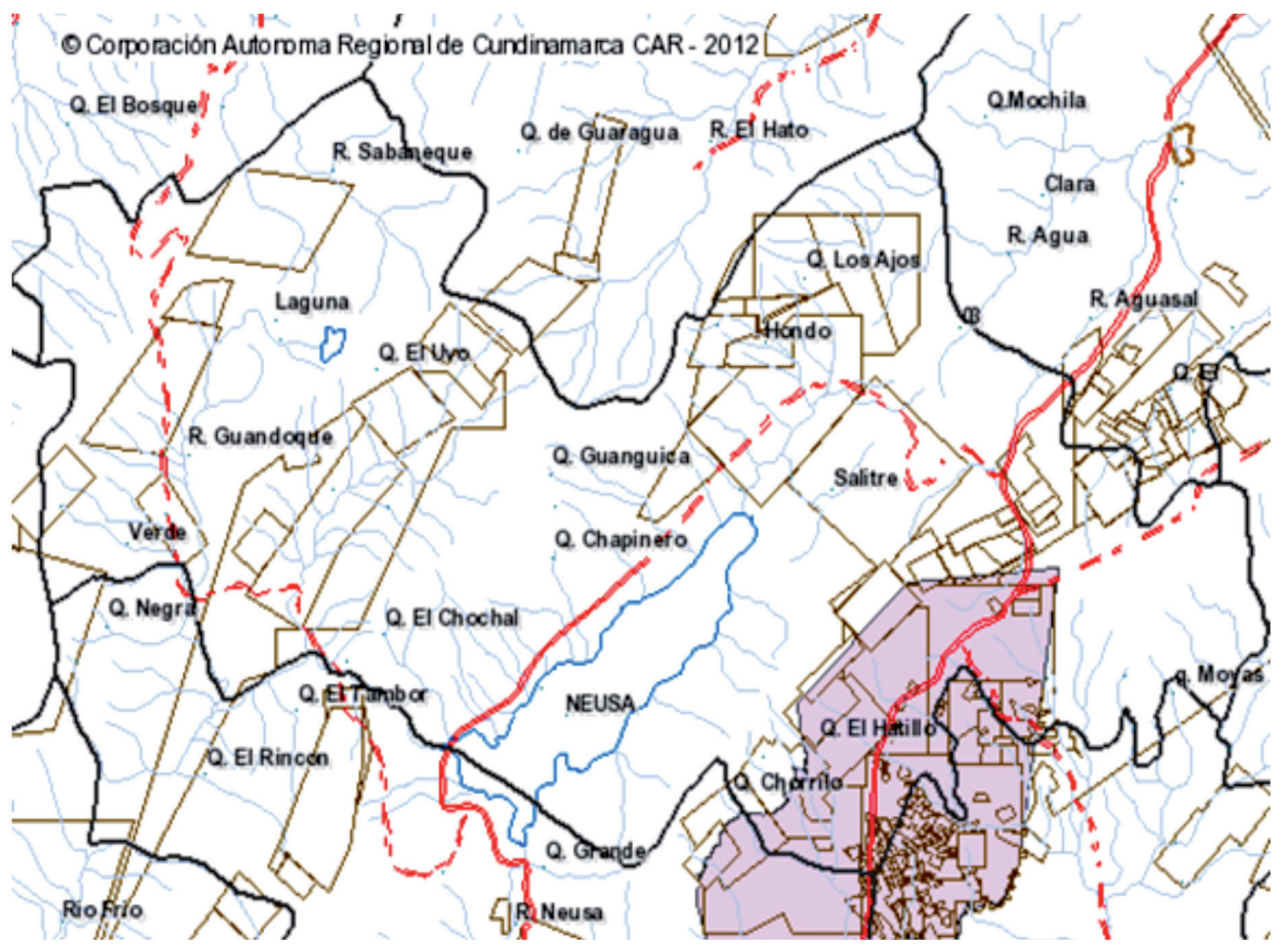

Figura 2. Mapa del municipio de Tausa. En líneas color marrón se demarcan títulos mineros vigentes al 2015. Las áreas compatibles con la minería según Resolución 222 de 1994 están sombreadas con color lila

Fuente: Corporación Autónoma Regional, 2012 [14].

entidades de control -ambiental y de salud pública-, prensa y academia»; y después de otras latitudes - Elsevier, Science Direct, Scopus(véase Tabla 1). Es oportuno señalar que para el caso colombiano se encontró mayor número de estudios de eventos de afectación al agua y al aire, siendo menor el número de artículos originales sobre afectación del recurso suelo por actividades de minería, respaldados en estudios analíticos [30].

Riesgos de afectación del suelo por extracción de carbón. En sendos estudios desarrollados en China y los países que lideran la producción de carbón se reporta la materialización de riesgos de contaminación física y química del suelo en terrenos que rodean minas subterráneas de carbón. Así, al norte de Bangladesh, donde colindan áreas mineras y de producción agrícola, se encontró en el suelo acumulación a niveles tóxicos de manganeso, zinc y plomo. Los índices de geoacumulación fueron: $I_{\text {geo }} \mathrm{Mn}$ $=(1,24 \pm 0,38), l_{\text {geo }} Z n=(1,49 \mathrm{ppm} \pm 0,58)$ y $l_{\text {geo }}$ $\mathrm{Pb}=(1,63 \mathrm{ppm} \pm 0,38),[24]$, cabe recalcar que estos valores corresponden a los índices de geoacumulación $\left[I_{\text {geo }}\right]=\log _{2}\left(C_{n} / 1.5 \mathrm{Bn}\right)$, donde $C_{n}$ es la concentración actual del elemento y $B_{n}$ la concentración evidenciada antes de 
Tabla 1. Eventos de degradación física y química del suelo relacionados con extracción y transformación de carbón y arcilla más reportados en la literatura científica

\begin{tabular}{|c|c|c|c|c|}
\hline \multirow[b]{2}{*}{ Evento no deseado } & \multicolumn{4}{|c|}{ Actividad / Ocurrencia de eventos no deseados } \\
\hline & $\begin{array}{l}\text { Extracción } \\
\text { subterránea de } \\
\text { carbón }\end{array}$ & $\begin{array}{l}\text { Extracción de } \\
\text { arcilla }\end{array}$ & $\begin{array}{l}\text { Fabricación de } \\
\text { ladrillos }\end{array}$ & $\begin{array}{l}\text { Obtención de coque } \\
\text { de carbón }\end{array}$ \\
\hline $\begin{array}{l}\text { Contaminación del suelo con } \\
\text { metales: } \mathrm{Cd}, \mathrm{Mn}, \mathrm{Pb} \text { y } \mathrm{Zn}\end{array}$ & Frecuente $^{\text {h, }}$ & Posible & Posible & Frecuente $^{\mathrm{b}}$ \\
\hline $\begin{array}{l}\text { Contaminación del suelo por } \\
\text { acumulación de ceniza, escoria } \\
\text { y polvo }\end{array}$ & Frecuente & Posiblei & Frecuente $^{c}$ & Frecuente $^{c}$ \\
\hline $\begin{array}{l}\text { Contaminación del suelo por } \\
\text { infiltración de efluentes }\end{array}$ & Frecuente & Poco probable & Poco probable & Poco probable \\
\hline $\begin{array}{l}\text { Contaminación del suelo por } \\
\text { lixiviados del material estéril }\end{array}$ & Posible f,g & Posible $^{f}$ & Posible $^{f}$ & Posible $e^{e, f}$ \\
\hline Deforestación & Frecuente $^{\mathrm{a}}$ & Frecuente ${ }^{j}$ & Frecuente & Frecuente \\
\hline Desestabilización del suelo & Frecuente $^{a}$ & Frecuente ${ }^{j}$ & Poco probable & Poco probable \\
\hline Deterioro estético de los suelos & Frecuente $^{\text {a }}$ & Frecuente a,j & Posible & Frecuente ${ }^{b}$ \\
\hline $\begin{array}{l}\text { Deterioro del paisaje por } \\
\text { abandono y acumulación de } \\
\text { residuos }\end{array}$ & Posible $^{a}$ & Posible $e^{a, j}$ & Frecuente $^{e}$ & Posible $^{b}$ \\
\hline Drenaje ácido de minas & Posible ${ }^{g}$ & Poco probable & Poco probable & Poco probable \\
\hline Erosión hídrica de los suelos & Frecuente $^{\mathrm{a}}$ & Frecuente $^{d}$ & Poco probable & Poco probable \\
\hline $\begin{array}{l}\text { Infiltración en el suelo de aguas } \\
\text { residuales domésticas. }\end{array}$ & Posible & Posible & Posible & Posible \\
\hline
\end{tabular}

Notas: aSánchez Rojas, 2008 [17], Concejo Municipal de Tausa, 2012 [13], ' Liu \& Yuan, 2016 [18], Nijhuis, 2014 [19], 'Bryers, 1996 [20], `Instituto Geográfico Agustín Codazi, 1997 [10], e Procuraduría General de la Nación, 2005[21], 'Ministerio de Minas y Energía \& Ministerio del Medio Ambiente, 2002 [22], ${ }^{8}$ Fierro Morales, 2012 [23], ' Bhuiyan, Parvez, Islam, Dampare, \& Suzuki, 2010 [24], Fan, Sun, Li, Zhao, Tian, \& Shao, 2013 [25], Jiang, Lu, Zhao, Yang, \& Yang, 2014 [26], 'Elmes, 2015 [27], ' Lozano Zafra \& Fierro Morales, 2008 [28], Guerrero Useda, 2014 [29].

Fuente: Elaboración propia.

establecerse una actividad antrópica, esto en ambientes no contaminados. Del mismo modo, en estudio desarrollado en los alrededores del vertedero de una mina subterránea de carbón en China, se constató la contaminación del suelo con $\mathrm{Zn}, \mathrm{Cd}$ y $\mathrm{Pb}$, la disminución gradual de su concentración al alejarse del vertedero, manteniendo niveles tóxicos a distancias de 500 metros [25], además se determinó que la mayor tasa y aceleración de acumulación la 
tiene el cadmio, por lo que a este metal se le atribuye el mayor riesgo de contaminación del suelo [26].

Otro riesgo de degradación física del suelo, relacionado con la minería subterránea de carbón, es la subsidencia del terreno por extracción de fluidos, por galerías mineras antitécnicas y por compactación del suelo. En el Plan para la Gestión del Riesgo del municipio de Tausa se reconoció el peligro de subsidencia y la necesidad de evitar la confluencia de usos de suelo para vivienda y minería. La literatura científica reporta fenómenos de subsidencia en minas subterráneas de carbón, los que han causado daño estructural a edificaciones próximas a los emplazamientos mineros. Al respecto ver: López Gayarre et al. (2010) [31], Giraldo \& Blas (2007) [32], Matos de la Torre (2014) [33]. Con todo, la evidencia técnica de la ocurrencia de este fenómeno en minas colombianas se limita a casos como el hundimiento del lecho del río Meléndez en inmediaciones de tres bocatomas del título minero CBR-101, localizado en el corregimiento de La Buitrera del municipio de Santiago de Cali. En este caso las labores extractivas fueron suspendidas [34].

El Plan para la Gestión del Riesgo del municipio de Tausa reportó la materialización de otros riesgos físicos del recurso suelo: se trata de la desestabilización de taludes en las quebradas Chorrillo y Hatillo a causa de vertimiento de estériles y residuos sólidos de la actividad minera arrojados en estas áreas [35].

Riesgos al suelo por extracción de arcilla. Lozano Zafra \& Fierro Morales (2008) [28], Granadillo Mendoza \& García Montesinos (2010) [36], Guerrero Useda (2014) [29], por citar algunos, reportaron la materialización de riesgos físicos de afectación al suelo en zonas de extracción de arcilla: procesos avanzados de degradación, deforestación y deterioro estético del suelo, así como deterioro del paisaje por abandono y acumulación de residuos en suelos aledaños a minas de arcilla. En la sabana de Bogotá son críticos los casos de Ciudad Bolívar y de los cerros aledaños a la laguna La Herrera. Así, en varios puntos de Ciudad Bolívar -hoy zona urbana del Distrito Especial de Bogotá-, donde se extraía arcilla, se incrementaron los fenómenos de remoción en masa y otros impactos sobre el suelo cuya reparación superará significativamente lo recaudado en su momento por regalías [28]. Es crítico el deterioro de los cerros que rodean la laguna La Herrera, ecosistema tipo Ramsar ubicado al sur de la sabana de Bogotá, donde además de la visible fractura del paisaje, la extracción de arcilla amenaza la biodiversidad por disminución de especies vegetales y animales propias de estos ecosistemas.

La degradación biológica del suelo en zonas de explotación minera se observa en la disminución de contenido de materia orgánica [37-38] y la subsiguiente disminución de la meso fauna edáfica. Este fenómeno fue constatado en la cantera Soratama, localizada en los cerros nororientales de Bogotá. El suelo de esta cantera, después de terminar las labores extractivas, debió someterse a enmienda para lograr el repoblamiento de meso fauna edáfica [39]. Para el contexto internacional se encontró referencia a este evento en Castellón, España, donde se extrae arcilla para la fabricación de cerámicas. Allí se desarrolló un análisis de ciclo de vida para el proceso de extracción y se determinó que en las labores de excavación, carga y transporte a las instalaciones de trituración y acopio, se usan maquinarias que funcionan con combustibles, por lo que además de los impactos físicos al suelo, estas labores 
generan emisiones contaminantes de consideración [40].

Riesgos de afectación del suelo por transformación de arcilla y carbón. Tanto los hornos utilizados para cocinar el ladrillo, como los empleados en la coquización de carbón generan polvo, humo, escoria y cenizas volátiles del carbón que por lixiviación de metales pesados presentes en estas, llegan a contaminar el suelo y las aguas subterráneas [41], por lo que sorprende que ni en los planes de desarrollo del municipio de Tausa, ni en el plan de gestión de riesgo, se reportara esta silenciosa amenaza que, además, produce serias afectaciones sobre la salud de los trabajadores y los habitantes de las zonas próximas a los hornos. Se encontró, en cambio, que la Procuraduría General de la Nación, tras visitar en 2005 las plantas de coquización de carbón del municipio de Tausa, constató altos niveles de contaminación atmosférica y reportó en su informe los siguientes eventos: alteración drástica del paisaje, un incendio forestal, una infiltración de lixiviados, hornos con emisiones altas de monóxido de carbono, óxidos de azufre y de nitrógeno, humo, hollín y ceniza [21]. Una situación comparable se registró en la vereda El Mochuelo Bajo de Ciudad Bolívar, donde mediante el análisis de imágenes satelitales se observó un área de $57.609 \mathrm{~m}^{2}$ con vegetación y viviendas cubiertas de hollín y cenizas de color negro, emanadas de los hornos a base de carbón utilizados para la cocción del ladrillo [42]; es propio indicar que en este municipio también concurren la minería de arcillas a cielo abierto y la elaboración de ladrillos y bloques con la arcilla extraída.

De los riesgos de absorción de contaminantes químicos en suelos mineros. Con el propósito de explorar la existencia de condiciones propicias para la acumulación de contaminantes químicos en el suelo, y sus posibles vías de movilización, se diseñó y desarrolló un muestreo al azar en suelos externos de un título minero (mina subterránea de carbón). Por tratarse de áreas de influencia industrial se tomaron muestras compuestas en 16 sitios; en las Tablas 2, 3, 4 y 5 se reporta el $\mathrm{pH}$, conductividad eléctrica y porcentaje de materia orgánica obtenido para cuatro muestras compuestas del suelo estudiado.

Lo obtenido para el $\mathrm{pH}$ de las muestras de los sitios 1, 2, 3 y 4, según parámetros del Departamento de Agricultura de los Estados Unidos (USDA), corresponde a un suelo extremadamente ácido. Estos datos están dentro de los rangos obtenidos por el Instituto Minero y Geológico de España (2002) [43], para suelos de la unidad tipo 1 (cuyo pH está entre 3,45 y 8,64 y contienen un porcentaje bajo de materia orgánica: 3,85\%). La calidad ácida de las muestras 1, 2, 3 y 4, de cara a lo establecido por Galán Huertos \& Romero Baena (2008) [44], quienes encontraron que la mayoría de los metales tienden a estar más disponibles a pH ácido, permite predecir la existencia de condiciones favorables para la acumulación de metales pesados en los sitios muestreados. Del mismo modo, los suelos con muy bajos valores de $\mathrm{pH}$ pueden alcanzar rápidamente límites tóxicos que dañan las raíces de las plantas [45]. Asimismo, los resultados obtenidos tienen coherencia con lo estipulado por el IGAC, haciendo referencia que aproximadamente en el $85 \%$ del territorio nacional los suelos poseen valores de $\mathrm{pH}$ menores a 5,5.

Los suelos muestreados mostraron valores menores de $1 \mathrm{dS} / \mathrm{m}$ de conductividad eléctrica, lo que los clasifica como no salinos [46]. Según la EPA y el IGAC, el carácter no salino obtenido en las muestras 1, 2, 3 y 4 permite 
Tabla 2. Análisis de pH según parámetros EPA y el IGAC para 4 muestras de suelo

\begin{tabular}{|c|c|c|c|c|c|c|}
\hline \multirow{2}{*}{ Parámetro } & \multirow{2}{*}{ Variable } & \multirow{2}{*}{ Dimensión } & \multirow{2}{*}{ No. } & \multirow{2}{*}{ Indicador } & \multicolumn{2}{|c|}{ Clasificación } \\
\hline & & & & & EPA & IGAC \\
\hline \multirow{4}{*}{ Ecológico } & \multirow{4}{*}{ Suelo } & \multirow{4}{*}{$\mathrm{pH}$} & 1 & 4.12 & $\begin{array}{c}\text { Extremadamente } \\
\text { ácido }\end{array}$ & $\begin{array}{c}\text { Extremadamente } \\
\text { ácido }\end{array}$ \\
\hline & & & 2 & 4.17 & $\begin{array}{c}\text { Extremadamente } \\
\text { ácido }\end{array}$ & $\begin{array}{c}\text { Extremadamente } \\
\text { ácido }\end{array}$ \\
\hline & & & 3 & 4.59 & $\begin{array}{c}\text { Extremadamente } \\
\text { ácido }\end{array}$ & $\begin{array}{l}\text { Muy fuertemente } \\
\text { ácido }\end{array}$ \\
\hline & & & 4 & 4.06 & $\begin{array}{c}\text { Extremadamente } \\
\text { ácido }\end{array}$ & $\begin{array}{c}\text { Extremadamente } \\
\text { ácido }\end{array}$ \\
\hline
\end{tabular}

Tabla 3. Conductividad eléctrica para 4 muestras de suelo y clasificación según la EPA y el IGAC

\begin{tabular}{|c|c|c|c|c|c|c|}
\hline \multirow{2}{*}{ Parámetro } & \multirow{2}{*}{ Variable } & \multirow{2}{*}{ Dimensión } & \multirow{2}{*}{ No. } & \multirow{2}{*}{ Indicador } & \multicolumn{2}{|c|}{ Clasificación } \\
\hline & & & & & EPA & IGAC \\
\hline \multirow{4}{*}{ Ecológico } & \multirow{4}{*}{ Suelo } & \multirow{4}{*}{$\begin{array}{l}\text { Conductividad } \\
\text { eléctrica } \\
\text { (mmho/cm) }\end{array}$} & 1 & 0.38 & $\begin{array}{c}\text { No } \\
\text { salino }\end{array}$ & Normal \\
\hline & & & 2 & 0.64 & $\begin{array}{c}\text { No } \\
\text { salino }\end{array}$ & Normal \\
\hline & & & 3 & 0.63 & $\begin{array}{c}\text { No } \\
\text { salino }\end{array}$ & Normal \\
\hline & & & 4 & 1 & $\begin{array}{c}\text { No } \\
\text { salino }\end{array}$ & Normal \\
\hline
\end{tabular}

Tabla 4. Porcentaje de carbono orgánico en 4 muestras de suelo y clasificación según la EPA

\begin{tabular}{|c|c|c|c|c|c|}
\hline \multirow[b]{2}{*}{ Parámetro } & \multirow[b]{2}{*}{ Variable } & \multirow[b]{2}{*}{ Dimensión } & \multirow[b]{2}{*}{ No. } & \multirow[b]{2}{*}{ Indicador } & Clasificación \\
\hline & & & & & EPA \\
\hline \multirow{4}{*}{ Ecológico } & \multirow{4}{*}{ Suelo } & \multirow{4}{*}{$\begin{array}{c}\text { Carbono } \\
\text { orgánico } \\
(\%)\end{array}$} & 1 & 3.7 & Alta \\
\hline & & & 2 & 6.0 & Muy alta \\
\hline & & & 3 & 1.1 & Baja \\
\hline & & & 4 & 0.5 & Muy baja \\
\hline
\end{tabular}


Tabla 5. Porcentaje de materia orgánica para 4 muestras de suelo y clasificación EPA e IGAC

\begin{tabular}{|c|c|c|c|c|c|c|}
\hline \multirow{2}{*}{ Parámetro } & \multirow{2}{*}{ Variable } & \multirow{2}{*}{ Dimensión } & \multirow{2}{*}{ No. } & \multirow{2}{*}{ Indicador } & \multicolumn{2}{|c|}{ Clasificación } \\
\hline & & & & & EPA & IGAC \\
\hline \multirow{4}{*}{ Ecológico } & \multirow{4}{*}{ Suelo } & \multirow{4}{*}{$\begin{array}{l}\text { Materia } \\
\text { orgánica }\end{array}$} & 1 & 6.4 & Orgánico & Medio \\
\hline & & & 2 & 10.4 & Orgánico & Alto \\
\hline & & & 3 & 1.9 & Mineralizado & Bajo \\
\hline & & & 4 & 0.9 & Muy mineralizado & Bajo \\
\hline
\end{tabular}

describir que esta zona no está propensa a facilitar la movilización y retención de metales pesados, por tanto se podría concluir que la actividad antrópica del sitio no está afectando el desarrollo de la biota que lo rodea. De otra parte, es importante tener en cuenta que la zona estudiada está entre una altura de 2.800 y 3.000 msnm (páramo), por lo que el suelo estudiado tendría que presentar un rango menor a $4 \mathrm{dS} / \mathrm{m}$ en la conductividad eléctrica.

Las muestras de suelos estudiados 1 y 2 se encuentran dentro de un rango alto y muy alto de carbono orgánico, lo cual podría favorecer la distribución del espacio poroso del suelo, y afectar diversas propiedades físicas, como la humedad aprovechable, la capacidad de aire y el movimiento de agua y gases del suelo; asimismo, puede intervenir en las propiedades biológicas, actuando como fuente energética para los organismos heterótrofos, lo que es un factor primordial para la productividad del mismo [47]. Sin embargo, las muestras 3 y 4 se encuentran dentro de un rango bajo y muy bajo de carbono orgánico, lo cual según [47], puede generar grandes cambios en la capacidad tampón del suelo.

Por otro lado, teniendo en cuenta la clasificación de materia orgánica, según la EPA, las muestras
1 y 2 se clasifican en orgánico; sin embargo, según el IGAC, estas se encuentran dentro del rango medio y alto, respectivamente. Estos suelos pueden presentar residuos orgánicos de origen animal o vegetal, que se encuentran en descomposición, y que se acumulan tanto en la superficie como dentro del perfil del suelo [47]. Asimismo, contribuye de modo importante al crecimiento de la vegetación, al proporcionar elementos esenciales como nitrógeno, fósforo y azufre; gracias a esta propiedad, la MOS ayuda a evitar la desecación y contracción en suelos arcillosos, así como mantiene la humedad en suelos arenosos [48]. Las muestras 3 y 4 se clasifican en un rango muy mineralizado, esto se da cuando los suelos son tóxicos para los microorganismos, que son los encargados de estimular la estructura adecuada del suelo, además de regular los cambios de $\mathrm{pH}$. Debido a que las muestras 3 y 4 no tienen buenas cantidades de materia orgánica, se puede deducir que contienen bajos niveles de nitrógeno, fósforo y azufre, encargados del crecimiento de la vegetación, pues sirve como energía para los organismos de la microflora y microfauna del suelo, lo que fomenta buena estructura [49].

Los organismos del suelo crecen mejor en un medio casi neutro. Según los parámetros 
obtenidos para la zona del estudio, se prevé la inhibición del crecimiento de la mayoría de los organismos, incluidas muchas bacterias y gusanos de tierra. El sueloácido retarda muchas actividades importantes llevadas a cabo por los microbios del suelo, incluida la fijación de nitrógeno, nitrificación y la descomposición de la materia orgánica [50].

Tras la determinación de las amenaza de contaminación del recurso suelo según el tipo de actividad antrópica, se pueden hacer diferentes estimaciones. El riesgo resulta de computar la amenaza y la vulnerabilidad que es el producto entre un índice «susceptibilidad/resiliencia» y una probabilidad «de exposición». Es necesario anotar que, en el ámbito del riesgo ambiental del suelo, la resiliencia resulta ser la capacidad del suelo para recuperarse, mientras que la susceptibilidad muestra la fragilidad del suelo. Sin embargo, la estimación de la resiliencia del suelo impone conocer índices más elaborados, como son los de geoacumulación y de carga de contaminación, cuyo cálculo demanda el acceso a datos sobre porcentaje de acumulación de los diferentes compuestos en muestras de suelo contaminadas y no contaminadas, lo que estuvo por fuera del alcance de este estudio. Se puede, de otra parte, acudir a estimaciones de la vulnerabilidad sobre escalas cualitativas, como se reporta en varios trabajos [51-52].

Puesto que para la extracción y transformación de carbón, procesos que confluyen en Rasgatá Bajo, se encontraron en la literatura científica reportes de ocurrencia de contaminación del suelo con metales ( $\mathrm{Cd}, \mathrm{Mn}, \mathrm{Pb}$ y $\mathrm{Zn}$ ), deforestación y deterioro estético del suelo, se desarrolló un análisis más detallado de estos riesgos. Para tener una primera aproximación a la magnitud del riesgo se trabajó en una escala que permitiera calificar la amenaza y la exposición como: reducida, moderada, alta y muy alta, asociándoles valores de $1 / 4,1 / 2,3 / 4$ y 1 , respectivamente [53]. En cuanto a la relación entre susceptibilidad y resiliencia, al tratarse de suelos ubicados a más de 2.500 msnm, en un área estratégica para la preservación de las fuentes hídricas, y que han estado expuestos por varios años a los efectos adversos de la explotación y trasformación de carbón, en el marco de una frágil institucionalidad ambiental y de escasez de recursos para la implementación de programas de diagnóstico y tratamiento del suelo, se calificó la susceptibilidad del de Rasgatá Bajo como muy alta, mientras la resiliencia muy baja $(\rightarrow 0)$. En este escenario, el riesgo de contaminación del suelo por acumulación de metales pesados alcanza calificaciones muy altas. Al acudir a una adaptación del método $\mathrm{POSH}$ para la caracterización de la carga contaminante sobre el suelo, fue posible asociar la posibilidad de presencia de una sustancia contaminante de este a un tipo de actividad antrópica (Tabla 1) y estimar la probable sobrecarga del suelo sobre la base de la confluencia de actividades de alto impacto.

En la Tabla 6 se muestra la forma en que se organizó la información para construir las diferentes hipótesis sobre fuente, tipo de contaminante y magnitud del riesgo de afectación al suelo.

Para evaluar el riesgo de contaminación del suelo por metales pesados, mediante la técnica de fluorescencia de rayos $X$ se estableció la composición de las muestras tomadas en el sitio más cercano a la boca de la mina Los Ocales, a una profundidad de 20 cm. Los resultados que se presentan en la Tabla 7 señalan un suelo rico en óxido de silicio, condición que debe investigarse a profundidad para efectos de establecer el impacto de este nutriente en la disponibilidad de metales. Para 
el $\mathrm{Pb}$ y $\mathrm{Zn}$ se observan concentraciones en los límites superiores de la media, que deben tratarse para reducir la vulnerabilidad del suelo.

Teniendo en cuenta los resultados obtenidos en la determinación de metales pesados, se puede observar que el suelo estudiado presenta indicios de contaminación de elementos traza, como $\mathrm{Pb}$, Cd, Cu y Zn, y $\mathrm{SiO}_{2^{\prime}}$ encontrándose en concentraciones por debajo de la normatividad internacional EPA, que al encontrarse biodisponibles en la zona pueden derivar en geoacumulación o en movilización hacia las personas cercanas y ocasionar el riesgo latente e intoxicación por la exposición continua a altas concentraciones de estos elementos. Obtener $I_{\text {geo }}$ similares a los determinados por Bhuiyan y otros (2010) [24] para el Mn, supone una concentración 5,2 veces menor a las 13 ppm evidenciadas en la boca de la mina Los Ocales de Tausa.

Encuandoalostiposyfuentes decontaminación secundaria y terciaria, se estableció que en las faenas de extracción y de transformación de arcillas y carbón son generados residuos

Tabla 6. Estimación de la magnitud del riesgo de afectación del recurso suelo por confluencia de eventos adversos relacionados con la explotación y coquización de carbón

\begin{tabular}{|c|c|c|c|c|}
\hline Evento & P(Amenaza) & $\begin{array}{c}\text { [Susceptibilidad]/ } \\
\text { [Resiliencia] }\end{array}$ & P (Exposición) & Riesgo \\
\hline $\begin{array}{c}\text { Contaminación del suelo con } \\
\text { metales: Cd, Mn, Pb y Zn. }\end{array}$ & Muy alta & $>1$ & Muy alta & Muy alto \\
\hline Deforestación & Muy alta & $>1$ & Muy alta & Muy alto \\
\hline Deterioro estético de los suelos & Alta & $>1$ & Muy alta & Muy alto \\
\hline $\begin{array}{c}\text { Deterioro del paisaje por } \\
\text { abandono y acumulación de } \\
\text { residuos }\end{array}$ & Alta & $<1$ & Alta & Alto \\
\hline $\begin{array}{c}\text { Infiltración en el suelo de aguas } \\
\text { residuales domésticas }\end{array}$ & Alta & $<1$ & Alta & Alto \\
\hline
\end{tabular}

Fuente: Elaboración propia, 2015.

Tabla 7. Resultados de fluorescencia de rayos $X$

\begin{tabular}{|c|c|}
\hline Elemento & Composición (ppm) \\
\hline $\mathrm{Pb}$ & 13 \\
\hline $\mathrm{Cd}$ & $<7$ \\
\hline $\mathrm{SiO}_{2}$ & 771 \\
\hline $\mathrm{Cu}$ & 14 \\
\hline $\mathrm{Zn}$ & 53 \\
\hline
\end{tabular}


sólidos cuyo abandono y acumulación, en el corto plazo, deterioran el paisaje, y a largo plazo contaminan el suelo. Considerando las condiciones existentes, otros riesgos actuales se identificaron en zonas de Tausa, donde se extraen y transforman minerales. Ciertamente, en áreas con carencia hídrica, como es el caso de las veredas Laderagrande y Rasgatá Bajo, se tiene previsto alimentar la infraestructura de riego con aguas extraídas de las minas [54]. Al estar este municipio, como la mayoría de los municipios del país, en mora de adoptar modernas plantas de tratamiento de aguas residuales no domésticas [55] hay probabilidad de que ocurran efectos con consecuencias graves sobre las cuencas y suelos.

\section{CONCLUSIONES}

La confluencia en la vereda Rasgatá Bajo de actividades de extracción y de transformación de carbón y arcilla incrementa el riesgo de degradación físico química del suelo. La extracción subterránea de carbón conlleva la generación de residuos sólidos y de aguas

Tabla 8. Modelo conceptual del riesgo de contaminación física y química del suelo por actividades de extracción y transformación de arcilla y carbón

\begin{tabular}{|c|c|c|c|c|c|c|}
\hline \multirow{2}{*}{$\begin{array}{l}\text { Tipo de } \\
\text { contaminación }\end{array}$} & \multirow{2}{*}{ Riesgo actual } & \multirow{2}{*}{ Mecanismo / Ruta } & \multicolumn{4}{|c|}{ Fuente de contaminación } \\
\hline & & & MC & MA & HA & $\mathrm{HC}$ \\
\hline \multirow{6}{*}{ 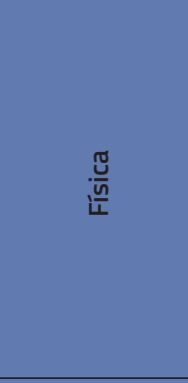 } & Degradación del suelo & & $x$ & $x$ & $x$ & $x$ \\
\hline & Deforestación & & $x$ & $x$ & $x$ & $x$ \\
\hline & Desestabilización del suelo & & $x$ & $x$ & & \\
\hline & Deterioro estético de los suelos & & $x$ & $x$ & $x$ & $x$ \\
\hline & $\begin{array}{l}\text { Deterioro del paisaje por abandono y acumulación de } \\
\text { residuos }\end{array}$ & & $x$ & $x$ & $x$ & $x$ \\
\hline & Erosión hídrica de los suelos & & $x$ & $x$ & & \\
\hline \multirow{6}{*}{ 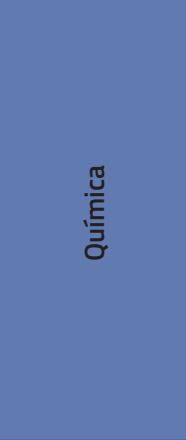 } & Contaminación del suelo con metales: Cd, Mn, Pb y Zn & $\begin{array}{l}\text { Precipitación/ } \\
\text { Acumulación/Viento }\end{array}$ & $x$ & $x$ & $x$ & $x$ \\
\hline & $\begin{array}{c}\text { Contaminación del suelo por acumulación de ceniza, } \\
\text { escoria y polvo }\end{array}$ & $\begin{array}{l}\text { Precipitación/ } \\
\text { Acumulación/Viento }\end{array}$ & $x$ & $x$ & $x$ & $x$ \\
\hline & Contaminación del suelo por infiltración de efluentes & Infiltración / Suelos & $x$ & $x$ & & \\
\hline & $\begin{array}{c}\text { Contaminación del suelo por lixiviados del material } \\
\text { estéril }\end{array}$ & Absorción / Aguas & $x$ & $x$ & $x$ & $x$ \\
\hline & Drenaje ácido de minas & Absorción / Aguas & $x$ & & & \\
\hline & Infiltración en el suelo de aguas residuales domésticas & Infiltración / Suelos & $x$ & $x$ & $x$ & $x$ \\
\hline
\end{tabular}

MC: mina de carbón, MA: mina de arcilla, HA: horno cocción de arcilla, HC: horno coquización de carbón.

Fuente: Elaboración propia, 2015. 
residuales domésticas y no domésticas que, al ser arrojados y vertidos sin tratamiento, contaminan el suelo, y que según sus grados de compactación, acidez, salinidad y mineralización son más proclives a acumular metales a niveles tóxicos para la flora, la fauna y las personas que habitan la zona.

Se encontró que, asociados a la extracción subterránea de carbón, hay al menos siete eventos adversos con ocurrencia frecuente, los que, a falta de prevención, monitoreo y oportuno tratamiento, hacen más vulnerable el territorio. Debido a que el suelo de Rasgatá Bajo está expuesto de manera simultánea a los efectos colaterales de la extracción y transformación del carbón, eventos como la contaminación del suelo con metales (Cd, Mn, Pb y Zn), la deforestación y el deterioro estético del suelo se hacen más riesgosos. Del mismo modo, por la concurrencia de procesos de transformación de arcilla y carbón, se incrementa el riesgo de contaminación del suelo por acumulación de cenizas y escoria, impacto que coincide con lo constatado en otros casos [42].

Finalmente, como salta a la vista, es necesario en estudios futuros determinar los tipos y concentraciones de metales pesados en muestras contaminadas y en no contaminadas del suelo de Rasgatá Bajo; estos análisis permitirán calcular, entre otros, los índices de carga de la contaminación y degeoacumulación. Con esta información podrán diseñarse planes pertinentes para el manejo ambiental y avanzar en la implementación de acciones de prevención y recuperación del suelo.

Se demostró que es posible avanzar en el diseño de modelos conceptuales de riesgo de contaminación del suelo, análisis que debe presidir el diseño y desarrollo de estudios cuantitativos para establecer la presencia, acumulación y vías de movilización de sustancias que podrían derivar en mayor degradación del suelo. Deben desarrollarse estudios que con referencia en datos epidemiológicos permitan valorar el riesgo de afectación de la salud a la que están expuestos los habitantes de la vereda por contaminación química del suelo.

\section{BIBLIOGRAFÍA}

[1] Garraín Cordero, D. (2009). Desarrollo y aplicación de las categorías de impacto ambiental. (Tesis para optar al título de doctor en Ingeniería del Producto y del Proceso). Universitat Jaume I.

[2] Mangena, S. \& Brent, A. (2006). Application of a Life Cycle Impact Assessment framework to evaluate and compare environmental performances with economic values of supplied coal products. Journal of Cleaner Production, 14, pp. 1071-1084.

[3] Chávez T. C., Castro L. J., Díaz-Barriga M. F. \& Monroy, F. M. (2011). Modelo conceptual de riesgo ambiental por arsénico y plomo en el distrito minero de Santa María de la Paz, San Luis Potosí, México. e-Gnosis, 9(1), pp. 1-25.

[4] Pérez-Martínez, I. \& Martín Romero, F. (2015). Uso de parámetros indirectos para la evaluación de la contaminación de suelos por metales pesados en una zona minera de San Luis Potosí, México. Boletín de la Sociedad Geológica Mexicana, 67(1), pp. 1-12.

[5] Pineda Acevedo, V. (2015). Evaluación de la contaminación del suelo por metales 
pesados producto de la actividad minera de carbón. Bogotá: Universidad El Bosque.

[6] Icontec. (2004). NTC 3656. Gestión ambiental. Suelo. Toma de muestras de suelo para determinar contaminación. Bogotá: Icontec.

[7] Icontec. (2008). NTC 5234. Calidad de suelo. Determinación de pH. Bogotá: Icontec.

[8] Icontec. (2008a). NTC 5349. Calidad de suelo. Determinación de las bases intercambiables: los cationes $\mathrm{Ca}, \mathrm{Mg}, \mathrm{Na}$. Bogotá: Icontec.

[9] Alcaldía de Tausa. (s.f.) (2015). Sitio web del municipio de Tausa. En: http://www. tausa-cundinamarca.gov.co/index. shtm|\#4 (23 de marzo de 2015).

[10] Instituto Geográfico Agustín Codazi. (1997). Plan de Ordenamiento Territorial del municipio de Tausa. Tausa: CAR-GTZ-KFW.

[11] Convenio Instituto Geográfico Agustín Codazzi - CAR - GTZ - KFW. (1998). Proyecto Checua. Guía simplificada para la elaboración del Plan de Ordenamiento Territorial municipal. Bogotá.

[12] Agencia Nacional de Minería. (2015). Caracterización de la actividad minera departamental. Departamento de Cundinamarca. Informe de fiscalización, Bogotá.

[13] Concejo Municipal de Tausa. (2012). Plan de desarrollo socioeconómico y medioambiental de Tausa 2012-2015. Tausa.

[14] Corporación Autónoma Regional. (2012). Cartografía. Bogotá.
[15] Olaya Bello, A. (enero de 2014). Diagnóstico general. Plan de Desarrollo Tausa. En: http://cundinet.cundinamarca. gov.co:8092/ entregas/TAUSA_Plan\%20 Desarrollo_2014_Enero/Diagnostico.pdf (20 de marzo de 2015)

[16] El Tiempo. (31 de agosto de 1996). Chircales, una sombra en la sabana. El Tiempo.

[17] Sánchez Rojas, W. A. (2008). Tausa. Plan de Desarrollo Municipal 2008-2011.

[18] Liu, X. \& Yuan, Z. (2016). Life cycle environmental performance of byproduct coke production. Journal of Cleaner Production, 112 (Part 2), pp. 1292-1301.

[19] Nijhuis, M. (2014). ¿Puede haber carbón limpio? National Geographic, 34(4), pp. 2451.

[20] Bryers, R. W. (1996). Fireside slagging, fouling, and high-temperature corrosion of heat-transfer surface due to impurities in steam-raising fuels. (E. Science, Ed.) Progress in Energy and Combustion Science, 22(1), pp. 29-120.

[21] Procuraduría General de la Nación. (2005). Procuraduría detecta contaminación atmosférica en proceso de destilación del carbón. Bogotá.

[22] Ministerio de Minas y Energía \& Ministerio del Medio Ambiente. (2002). Guía mineroambiental de explotación. Bogotá.

[23] Fierro Morales, J. (2012). Políticas mineras en Colombia. Bogotá: ILSA. 
[24] Bhuiyan, M. A., Parvez, L., Islam, M., Dampare, S. B. \& Suzuki, S. (2010). Heavy metal pollution of coal mine-affected agricultural soils in the northern part of Bangladesh. (E. B.V, Ed.) Journal of Hazardous Materials, 173(1), pp. 184-192.

[25] Fan, J., Sun, Y., Li, X., Zhao, C., Tian, D. \& Shao, L. (2013). Pollution of organic compounds and heavy metals in a coal gangue dump of the Gequan Coal Mine, China. Chinese Journal of Geochemistry, 32(3), pp. 241-247.

[26] Jiang, X., Lu, W. X., Zhao, H. Q., Yang, Q. C. \& Yang, Z. P. (2014). Potential ecological risk assessment and prediction of soil heavy-metal pollution around coal gangue dump. Natural Hazards and Earth System Sciences, 14(6), pp. 1599-1610.

[27] Elmes, N. (20 de enero de 2015). Environmentalists warn about coal ash in clay mines. McClatchy - Tribune Business News.

[28] Lozano Zafra, D. \& Fierro Morales, J. (2008). Aproximación a la cuantificación de pasivos ambientales relacionados con la minería en la localidad de Ciudad Bolivvar, Bogotá, D.C. mediante el análisis multitemporal de fotografías aéreas $e$ imágenes satelitales. Bogotá.

[29] Guerrero Useda, M. (2014). Minería de carbón en la sabana de Bogotá ¿podría ser sostenible? IIEC, 3(2), pp. 11-16.

[30] Camargo García, J. C., Arias Morales, J. P. \& Muñoz Paredes, D. (2015). Evaluación del contenido de mercurio en suelos y lechos de quebradas en la zona minera de Miraflores, Quinchía, Colombia. Acta agronómica, 64(2), pp. 165-177.

[31] López Gayarre, F., Álvarez-Fernández, M., González-Nicieza, C., Álvarez-Vigil, A. \& Herrera García, G. (2010). Forensic analysis of buildings affected by mining subsidence. Engineering Failure Analysis, 17(1), pp. 270-285.

[32] Giraldo, M. \& Blas, W. (2007). Minería actual del carbón en el norte del Perú. Revista del Instituto de Investigación de la Facultad de Ingeniería Geológica, Minera, Metalúrgica y Geográfica, pp. 76-81.

[33] Matos de la Torre, E. M. (2014). Caracterización hidrogeológica de la cuenca del río Turón (Asturias) en relación con la clausura de explotaciones mineras de carbón. (Tesis). Universidad de Oviedo, Oviedo, España.

[34] Ingeominas. (2009). Informe visita técnica corregimiento de La Buitrera, municipio de Santiago de Cali. Santiago de Cali.

[35] Alcaldía Municipal de Tausa. (2012). Decreto No. 068 de 2012. Tausa.

[36] Granadillo Mendoza, E. \& García Montesinos, M. (2010). Participación comunitaria y conservación ambiental en el ámbito rural del estado Lara, Venezuela. Milticiencias, 10(3), pp. 249-256.

[37] Tudeta Serrano, M. D. (1993). Estado actual de la degradación de suelos calizos en varias zonas de la región de Murcia. Murcia.

[38] Campillo, M., Martín, F., Simón, M. \& Iriarte, A. (2000). Cuantificación 
de las propiedades de los suelos en explotaciones mineras a cielo abierto. Edafología, 7-3, pp. 31-42.

[39] Granados-Hernández, A. \& BarreraCataño, J. (2007). Efectos de la aplicación de biosólidos sobre el repoblamiento de la mesofauna edáfica de la cantera Soratama, Bogotá, D.C. Universitas Scientiarum. Enero-junio de 2007, 12 (edición especial II), pp. 73-84.

[40] Bovea, M. D., Saura, Ú., Ferrero, J. L. \& Giner, J. (2007). Cradle-to-gate study of red clay for use in the ceramic industry. The International Journal of Life Cycle Assessment, 12(6), pp. 439-447.

[41] Moreno, N. (2009). Cenizas volátiles de la combustión del carbón y el coque de petróleo: posibles aplicaciones innovadoras y aplicaciones actuales. Waste Management \& Research, 27(10), pp. 976-987.

[42] Garzón Tovar, N. (2013). Análisis preliminar de los impactos ambientales y sociales generados por la minería de arcillas a cielo abierto en la vereda El Mochuelo Bajo de Ciudad Bolívar. Bogotá: Pontificia Universidad Javeriana.

[43] Instituto Minero y Geológico de España. (2002). Determinación de niveles de fondo y niveles de referencia de metales pesados y otros elementos traza en suelos de la Comunidad de Madrid. Madrid.

[44] Galán Huertos, E. \& Romero Baena, A. (2008). Contaminación del suelo por metales pesados. Revista de la Sociedad Española de Mineralogía, 10, pp. 48-60.
[45] Metrohm International Headwuarters. (2013). Valor pH, conductividad y titulación en el análisis de agua y suelo. Herisau, Suiza.

[46] Noguera, S.\&Armado, A. (2010). Evaluación de metales en suelos contaminados. Avances en Química, pp. 167-175.

[47] Martínez, E., Fuentes, J. P. \& Acevedo, E. (2008). Carbono orgánico y propiedades del suelo. Revista de la ciencia del suelo y nutrición vegetal, 8(1), pp. 68-96.

[48] Doménech, X. (1995). Química del suelo. Madrid.

[49] Bohn, H., McNeal, B. \& O'Connor, G. (1993). Química del suelo. México: Editorial Limusa.

[50] Plaster, E. (2000). La ciencia del suelo y su manejo. España: Paraninfo.

[51] Concejo Municipal de Tausa. (20122015). Por el cual se adopta el plan de desarrollo socioeconómico y medioambiental de Tausa. Tausa.

[52] Bravo Mendoza, Ó. \& Sánchez Celis, M. (2012). Gestión integral de riesgos. (Cuarta ed.). Bogotá: B\&S.

[53] Foster, S., Hirata, R., Gomes, D. \& Délia, M. P. (2003). Protección de la calidad del agua subterránea. Washington: Banco Mundial.

[54] Concejo Municipal de Tausa. (2000). Plan de Ordenamiento Territorial. Tausa.

[55] Unicef, Colombia. (2008). El agua potable y el saneamiento básico en los planes de desarrollo. En C. Unicef, La infancia, el agua y el saneamiento básico en los planes de desarrollo departamentales y municipales. Bogotá, pp. 31-56. 